\title{
Anabases
}

ANABASES Traditions et réceptions de l'Antiquité

$7 \mid 2008$

Varia

\section{Linda Jones Roccos, Ancient Greek Costume : An Annotated Bibliography, 1784-2005}

Adeline Grand-Clément

\section{OpenEdition}

\section{Journals}

Édition électronique

URL : http://journals.openedition.org/anabases/2584

DOI : 10.4000/anabases. 2584

ISSN : 2256-9421

\section{Éditeur}

E.R.A.S.M.E.

\section{Édition imprimée}

Date de publication : 1 mars 2008

Pagination : 305-307

ISSN : 1774-4296

\section{Référence électronique}

Adeline Grand-Clément, «Linda Jones roccos, Ancient Greek Costume : An Annotated Bibliography,

1784-2005 », Anabases [En ligne], 7 | 2008, mis en ligne le 01 décembre 2011, consulté le 22 septembre 2020. URL : http://journals.openedition.org/anabases/2584; DOI : https://doi.org/10.4000/anabases. 2584

Ce document a été généré automatiquement le 22 septembre 2020

(c) Anabases 


\title{
Linda Jones Roccos, Ancient Greek Costume: An Annotated Bibliography, 1784-2005
}

\author{
Adeline Grand-Clément
}

\section{RÉFÉRENCE}

Linda Jones Roccos, Ancient Greek Costume : An Annotated Bibliography, 1784-2005, Jefferson, McFarland and Co, 2006, $175 \mathrm{p}$.

39,95 dollars / ISBN 0-7864-2274-4.

1 L.J. Roccos a repris dans ce volume la version annotée de la bibliographie qu'elle propose en ligne (http://scholar.library.csi.cuny.edu/roccos/greekcostume/), en la complétant par trois courtes études introductives et un index. L'auteur, qui travaille au College of Staten Island Library de New York, a recensé six cent trois références d'études, parues entre 1784 et 1995 , qui traitent de la parure vestimentaire dans le monde grec, depuis la période néolithique jusqu'à l'époque romaine. Un tel instrument de travail a le mérite de citer non seulement les ouvrages et les articles de revues, mais aussi les chapitres de volumes et les notices d'encyclopédies, ainsi que les comptes rendus qui s'y rapportent. L.J. Roccos a opté pour un classement alphabétique, par nom d'auteur, ce qui rend le recours à l'index indispensable - une présentation thématique et/ou chronologique (telle qu'on la trouve en ligne, par exemple) aurait peut-être été plus judicieuse et utile.

2 L'inventaire bibliographique est précédé de trois essais, qui auraient mérité d'être plus détaillés et mieux structurés. Dans le premier (Introduction to the Subject of Ancient Greek Costume), l'auteur rappelle que l'histoire des vêtements constitue un bon indice des changements sociaux et économiques - on pourrait ajouter politiques - que connaissent les sociétés. L'importance des vêtements se mesure par exemple à l'aune de la place non négligeable qu'ils occupent dans les mythes grecs et dans les pratiques 
rituelles. Après avoir rappelé la simplicité des pièces de tissu qui servent à habiller le corps, en Grèce ancienne, et peuvent être arrangées de mille façons, l'auteur pointe certains "problèmes » qui surgissent lorsque l'on entreprend une étude générale du costume grec. Elle évoque ainsi la complexité de la terminologie, qui s'accorde mal avec les témoignages iconographiques, l'extrême diversité régionale et les évolutions chronologiques (mais en quoi constituent-elles un "problème»?). Dans le deuxième essai (Themes of Scholarly Interest), l'auteur établit un bref historique des points qui ont suscité l'intérêt des chercheurs jusqu'ici et montre que les études, d'abord tournées vers la description et la tentative de reconstitution, s'orientent désormais vers les questions d'interprétation. L'ouvrage de L. Cleland et H. Llewellyn-Jones (The Clothed Body in the Ancient World, 2005) symbolise à ses yeux une telle inflexion : l'habillement n'y est plus étudié comme un simple vestige de la culture matérielle, mais comme un moyen d'interroger la signification de l'acte qui consiste à "vêtir le corps", en le replaçant dans son contexte culturel, social et artistique. Ajoutons - l'auteur ne semble pas en avoir connaissance - que certains travaux de l'équipe Phéacie (Paris I et VII) rejoignent tout à fait ce type de problématique, en rattachant l'histoire du vêtement à l'anthropologie du corps: F. Gherchanoc et V. Huet y étudient les « pratiques politiques et culturelles du vêtement" en Grèce et à Rome. Enfin, L.J. Roccos souligne l'accroissement de nos connaissances en matière d'artisanat textile, grâce aux progrès de l'archéologie, ainsi que l'intérêt tout particulier porté au vêtement féminin - elle mentionne notamment le débat qui porte sur les korai de l'Acropole d'Athènes. Vient ensuite l'Introduction to the Annotated Bibliography, dernière mise au point introductive, qui prend la forme d'un bilan historiographique très succinct et qui recoupe en partie l'essai précédent - sans pour autant justifier le choix de la date retenue comme point de départ de la recension, 1784. L'auteur insiste sur la multiplication des études à partir de 1975 et attribue deux causes à un tel regain d'intérêt : la montée du féminisme et cette seconde explication nous semble beaucoup plus pertinente - un tournant sociologique et anthropologique dans les études classiques, qui invite à appréhender le vêtement comme un marqueur culturel. Parce que la parure vestimentaire renvoie aux rites religieux et aux structures sociales, elle constitue un facteur de différenciation au sein de la société et participe à la construction de l'identité grecque face aux Barbares. L'auteur aurait pu dire ici un mot de l'apport des recherches menées par Roland Barthes (auteur totalement absent de l'ouvrage) autour de la sémiotique du vêtement, plutôt que de se livrer à une brève revue des ressources électroniques disponibles, qui clôt l'introduction, en laissant un peu le lecteur sur sa fin.

La bibliographie proposée résulte d'un méticuleux travail de dépouillement. Les principaux éléments du costume grec ont été pris en considération, des coiffures aux chaussures, des pièces de tissu jusqu'aux bijoux. Les études recensées recoupent des sujets et des domaines variés : divinité et religion, statues et sculpture, travail du textile, théâtre et costumes, tradition vestimentaire des étrangers. Bien évidemment, la liste établie n'a rien d'exhaustif - et n'en a pas l'ambition : sur un terrain d'étude aussi vaste et aux frontières mouvantes, les sélections opérées sont forcément partiales et résultent d'intérêts personnels : on constate ainsi que la sculpture a été privilégiée au détriment de la peinture sur vase (le nombre d'entrées "statue ", dans l'index, dépasse celui des entrées "textile»!). Aucune étude de F. Lissarrague ni de F. Frontisi (le masque ayant pourtant à voir avec le vêtement) n'est mentionnée. On peut regretter également que certains points, qui présentent pourtant des affinités importantes avec les pratiques vestimentaires et la parure du corps, aient été laissés de côté : signalons le 
cas de la cosmétique et des parfums. Ajoutons que les annotations qui suivent les références présentent un intérêt inégal; certaines d'entre elles souffrent de minimalisme et d'une absence de problématisation: elles ne font pas ressortir le lien avec le vêtement, ni l'apport historiographique de l'étude.

4 L'index général, à la fin du volume, s'avère indispensable pour naviguer dans le répertoire bibliographique; il inventorie les thèmes, personnages, lieux, termes grecs (mais seulement ceux qui renvoient à des pièces du vêtement grec) traités dans les études. Relativement fourni, il aurait toutefois gagné à être encore plus étoffé et cohérent : on ne trouve aucune entrée à vase ou à vase-painting, ni à weaving; black n'y figure pas, à l'inverse de blue et white. Dans certains cas, lorsque les résultats sont nombreux, l'auteur aurait pu distinguer des sous-entrées afin de guider le lecteur.

5 Ces quelques réserves ne doivent pas faire oublier l'intérêt du volume, précieux pour tous les chercheurs actuellement intéressés par ce domaine en plein renouveau historiographique - archéologues, historiens, historiens de l'art, anthropologues, littéraires et philologues. Cet outil de travail devra toutefois être complété par une mise à jour régulière, car il risque de devenir très vite dépassé. L.J. Roccos, qui en est pleinement consciente, annonce qu'elle proposera régulièrement des mises à jour sur le site internet et invite les lecteurs à contribuer à cet enrichissement par leurs suggestions.

\section{AUTEURS}

\section{ADELINE GRAND-CLÉMENT}

Université de Toulouse II-Le Mirail

adelinegc@yahoo.fr 